\title{
Electronic structure of two interacting phosphorus $\delta$-doped layers in silicon
}

\author{
D. J. Carter, ${ }^{1,2}$ O. Warschkow ${ }^{3}$ N. A. Marks, ${ }^{1}$ and D. R. McKenzie $^{3}$ \\ ${ }^{1}$ Nanochemistry Research Institute, Curtin University, GPO Box U1987, Perth WA 6845, Australia \\ ${ }^{2}$ iVEC, 26 Dick Perry Avenue, Kensington WA 6151, Australia \\ ${ }^{3}$ Centre for Quantum Computation and Communication Technology, \\ School of Physics, The University of Sydney, Sydney NSW 2006, Australia
}

(Dated: December 21, 2012)

\begin{abstract}
Density functional theory is used to quantify the interaction of a pair of $1 / 4$ monolayer phosphorus $\delta$-doped layers in silicon. We investigate changes in the electronic structure as the distance between the two $\delta$-doped layers is altered and identify the onset of interactions between the transverse and longitudinal bands. The calculations show that the valley splitting is insensitive to the separation distance, while the interlayer band splittings are insensitive to the representation used to describe the dopant disorder. These observations are exploited in a hybrid model which enables the calculation of accurate splittings of realistically disordered systems at tractable computational cost.

PACS numbers: $61.72 . u f, 71.20 . \mathrm{Mq}, 71.20 . \mathrm{Nr}, 71.55 . \mathrm{Cn}$
\end{abstract}

\section{INTRODUCTION}

Scanning tunnelling microscopy (STM) lithography is emerging as a potent technology to pattern phosphorusin-silicon devices at the atomic scale. ${ }^{1,2}$ This technique has been employed to fabricate a number of Si:P nanoscale structures, including atomically sharp $\delta$-layers, ${ }^{3-7}$ atomically thin wires, ${ }^{8}$ few-atom quantum dots,${ }^{9}$ and a prototype single-atom transistor. ${ }^{10}$ These devices are essentially two-dimensional structures since the lithographically-positioned phosphorus atoms are confined to a single atomic plane and buried by silicon overgrowth. Three-dimensional structures, obtained by repeating the 2D-lithography/overgrowth process, offer the prospect of more complicated device structures in which two or more patterned Si:P layers interact. In this paper we use density functional theory (DFT) to examine the electronic structure of the simplest multi-layer structure, that is, two stacked Si:P $\delta$-layers. The fabrication of such structures is currently being explored ${ }^{11,12}$ and a key engineering question is the transition from electronically isolated to strongly interacting with distance.

The electronic structure of single Si:P $\delta$-layers has been extensively studied using a variety of theoretical approaches, including effective mass theory, ${ }^{13,14}$ a conduction band model, ${ }^{15}$ various levels of tight-binding, ${ }^{16-18}$ and full DFT. ${ }^{19-22}$ Each of these methods brings its own set of advantages and limitations: effective mass theory and tight-binding can handle the length scales required for device modelling, while DFT affords a more rigorous treatment on smaller scales. That said, the high cost of full DFT calculations invariably requires some form of balance to be struck between various computational parameters such as the number of atoms, degree of in-plane dopant disorder, k-point density, basis-set quality and choice of exchange-correlation functional. In our previous DFT calculations ${ }^{19,20}$ we employed a compact basis which allowed us to treat as many as 800 atoms and a variety of dopant arrangements and concentrations in the $\delta$-plane. An alternative approach by Drumm et al. ${ }^{21}$ focussed on basis-set convergence for a single, highlyordered 1/4 monolayer (ML) geometry. In a follow-up paper, ${ }^{22}$ this approach was applied to systems with phosphorus atoms in several adjacent atomic layers. In our previous studies we also assessed the merits of a mixedatom pseudopotential model in which atoms in the $\delta$ plane are described as fractionally intermediate between silicon and phosphorus. While the mixed-atom pseudopotential cannot describe the effect of explicit-atom dopant disorder on the valley splitting (see Fig. 12 in Ref. 20) it provides a straightforward and economical treatment that brings out the qualitative aspects of the electronic structure.

In this work we apply DFT to study pairs of interacting $\mathrm{Si}: \mathrm{P} \delta$-layers. Using the mixed-atom pseudopotential approach we describe the merging of the electronic band structure of two isolated $1 / 4 \mathrm{ML} \delta$-layers into a single $1 / 2 \mathrm{ML} \delta$-layer. Explicit-atom pseudopotentials are used to illustrate the effect of dopant placement on the interactions between layers. We relate our results to an effective mass treatment of a pair of $\delta$-layers ${ }^{23}$ and earlier work on Si:P superlattices. ${ }^{13,14}$ Using a hybrid approach that combines mixed and explicit-atom treatments we estimate accurate valley and interlayer band splittings for realistically disordered double $\delta$-layer systems.

\section{METHODOLOGY}

Two interacting $\delta$-doped layers in silicon are described using a highly asymmetric periodic superlattice, as illustrated in Fig. 1(a). As in our previous studies, ${ }^{19,20}$ the unit cell is highly elongated in the plane-perpendicular direction and exceedingly compact in the in-plane directions; the extreme length in the plane-perpendicular direction is required to fully separate the interacting $\delta$ layers from their periodic images. The typical $\delta$-doping concentration achieved following a phosphine saturation dose and thermal anneal ${ }^{3,4,24}$ is $1 / 4$ ML; various earlier studies $^{16,20,21}$ have shown that at least 80 atomic layers 


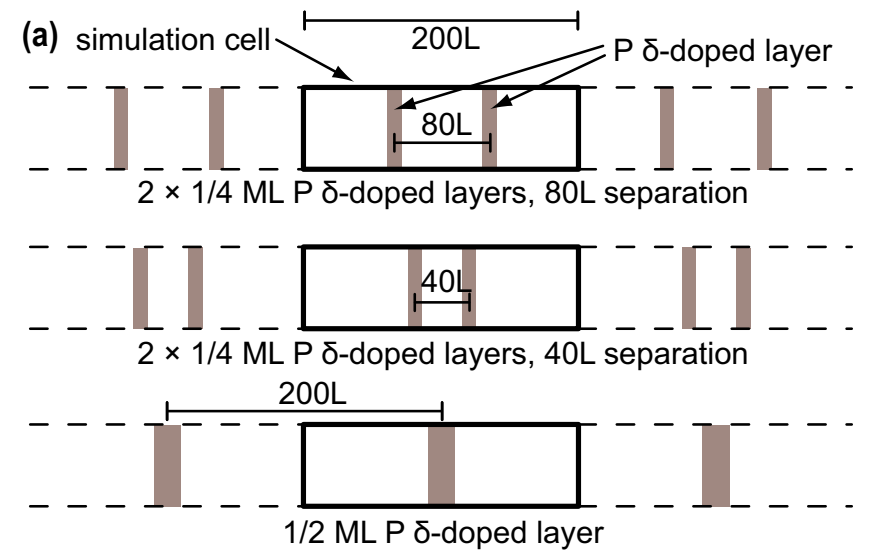

(b)

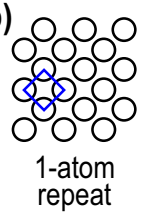

(c)

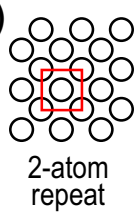

(d)

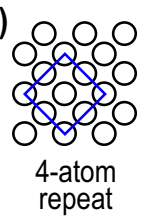

(e)
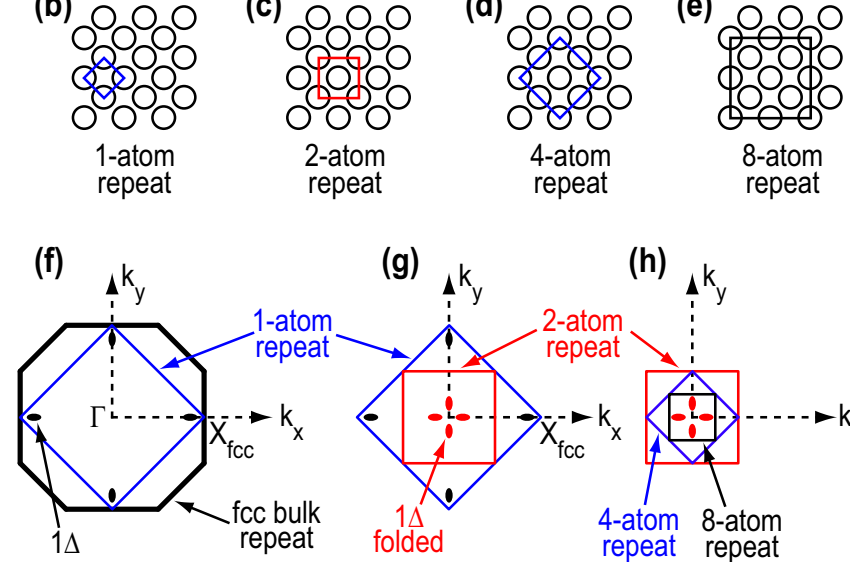

(g)

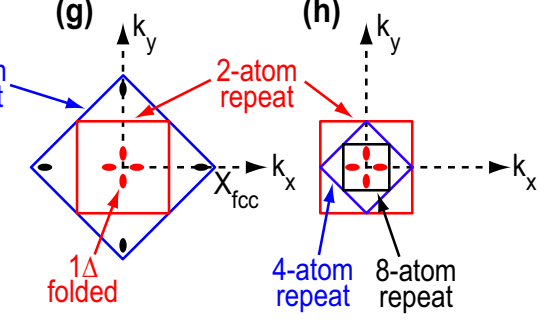

FIG. 1: Schematic illustration of the 3D periodic superlattices and reciprocal Brillouin zones used to represent two interacting $\delta$-layers. Panel (a) illustrates the separation dependence of $\delta$-layers that is explored in this work. The dimensions of the simulation cell (shown as a rectangular box with a solid outline) are exaggerated; in the actual cells the length is up to 50 times greater than the width. Panels (b-e) shows the various in-plane repeats used, containing between one and eight atoms in the unit cell. Panels ( $\mathrm{f}-\mathrm{h}$ ) shows the size and shape of the corresponding Brillouin zones in the $\mathrm{k}_{\mathrm{x}}, \mathrm{k}_{\mathrm{y}}$ plane, and their relation to the Brillouin zone of bulk fcc silicon. Small ellipsoids are used to indicate the position of the $1 \Delta$ conduction band minimum and its folding closer to the $\Gamma$ point for 2-, 4- and 8-atom repeats.

are required to provide an adequate degree of electronic separation for this concentration. The in-plane unit cell [Figs. 1(b-e)] has a square geometry and its size depends on how the phosphorus dopant distribution is represented as will be described below. In practical terms, our calculations utilize a total of between 80 and 960 atoms in the three-dimensional cell.

All calculations are performed using the SIESTA software. ${ }^{25}$ In the majority of cases the valence and conduction bands are expanded in a compact basis set of numerical atomic functions of single-zeta-plus-polarization (SZP) quality. A small number of double-zeta-pluspolarization (DZP) calculations were performed to as- sess the effects of a more complete basis. All basis functions are radially confined such that orbital energies are shifted by 0.01 Ry (see Ref. 25 for details). Core levels are represented using norm-conserving Troullier-Martins pseudopotentials. ${ }^{26}$

Electron exchange and correlation are treated using the generalized gradient approximation (GGA; PerdewBecke-Ernzerhof functional; Ref. 27) on a real-space mesh with a kinetic energy cutoff of 300 Ry. With these parameters the calculated silicon lattice constant is $5.58 \AA$ for an SZP basis and $5.49 \AA$ for a DZP basis; see Table I in Ref. 20. This compares favorably with the experimental value of $5.43 \AA$, keeping in mind the small systematic overestimate that is intrinsic to DFT-GGA. Earlier studies, ${ }^{19-21}$ including our own, have found that optimisation of the internal atomic coordinates has a minimal effect on the physical and electronic structure; hence all calculations are performed using bulk lattice positions. All band energies and the Fermi level $\left(\mathrm{E}_{\mathrm{F}}\right)$ are reported relative to the bulk conduction band minimum (CBM).

Dopant distributions in the $\delta$-plane are represented using two approaches: explicit dopant placement and mixed-atom pseudopotentials. ${ }^{28,29}$ The mixed-atom approach allows us to use in-plane repeats as small as one atom, whereas the explicit-atom treatment requires larger unit cells, depending on the dopant concentration and disorder. The majority of our calculations use the mixed-atom approach to describe two $1 / 4$ ML $\delta$-layers in a cell with a plane-perpendicular length of 200 atomic layers (denoted 200L). For 1- and 2-atom in-plane repeats [Figs. 1(b,c)], reciprocal space is sampled using $16 \times 16 \times 1$ and $10 \times 10 \times 1 \mathrm{k}$-grids, respectively, which are sufficiently converged for the purpose of this work. ${ }^{20}$ Convergence is assisted by the use of Methfessel-Paxton smearing (Ref. 30; polynomial of order $5, \mathrm{~T}=298 \mathrm{~K}$ ) which is employed for all systems considered. For calculations using explicit dopant representations we use 4- and 8-atom inplane repeats [Figs. 1(d,e)], employing k-point meshes of $8 \times 8 \times 1$ and $5 \times 5 \times 1$, respectively. The use of these larger in-plane unit cells necessitates a reduction of the planeperpendicular repeat from $200 \mathrm{~L}$ to $120 \mathrm{~L}$. For calculations involving only a single $\delta$-layer an $80 \mathrm{~L}$ cell is used. The use of larger in-plane repeats affects the bandstructure by progressively reducing the Brillouin zone as sketched in Figs. 1(f-h).

\section{RESULTS \& DISCUSSION}

\section{A. Single $\delta$-layers}

The starting point for understanding two interacting $\mathrm{Si}: \mathrm{P} \delta$-layers is the band structure of an isolated layer as discussed in detail in Refs. 15,17-21. The salient aspects are summarized in Fig. 2. The elongated unit cell used for the $\delta$-layer calculations causes the bulk silicon band structure to fold as shown in Fig. 2(a) when a 1-atom in-plane repeat is used. Here, the six conduction band 

1-atom $\times 80 \mathrm{~L}$ undoped

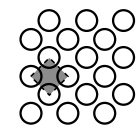
8-atom $\times 80 \mathrm{~L}$ undoped

\begin{abstract}
8 -atom $\times 80 \mathrm{~L}$ mixed-atom
\end{abstract}
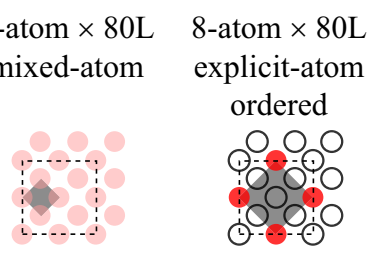
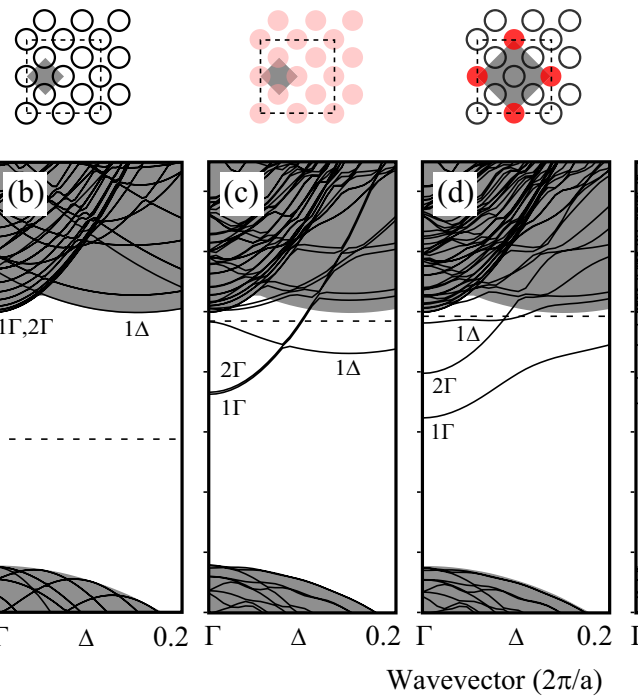

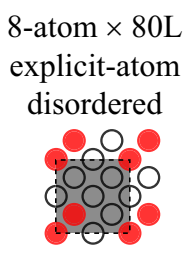

8 -atom $\times 80 \mathrm{~L}$ explicit-atom disordered
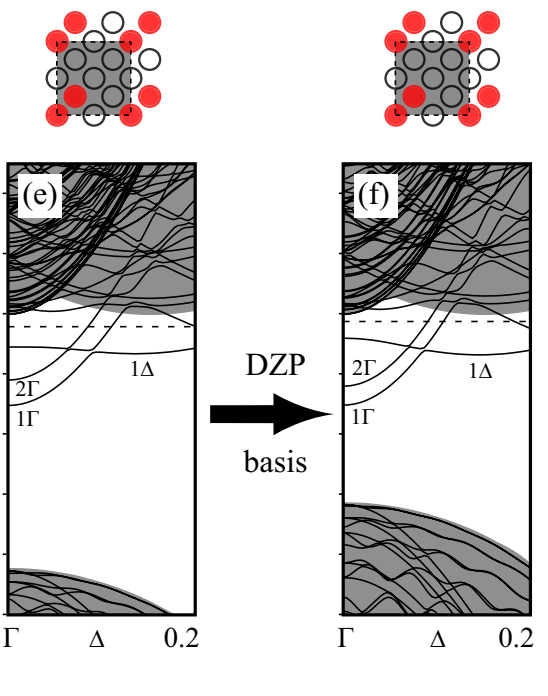

FIG. 2: Calculated band structure of (a,b) bulk silicon, and (c-f) a single 1/4 ML phosphorus $\delta$-layer in silicon for a variety of approximations used in this work. Schematic illustrations of the dopant plane are shown above the band structures, in which the dopant repeat pattern and the computational unit cell (which may be larger) have been outlined using gray background shading and dashed lines, respectively. The panels show $(\mathrm{a}, \mathrm{b})$ bulk silicon in 1- and 8-atom computational unit cells, (c) 1/4 ML mixed-atom doping, (d) 1/4 ML explicit dopants with a 4-atom dopant repeat, (e) 1/4 ML explicit dopants with a quasi-disordered 8-atom dopant repeat, and (f) the same as (e) but with a larger, more complete basis set. All calculations were performed using an 80L unit cell and an SZP basis set, with the exception of (f) which uses a DZP basis. The plane-projected bulk band structure of $\mathrm{Si}$ is represented by a gray continuum and the Fermi level is indicated by the dashed line. $\Gamma$ and $\mathrm{X}_{\mathrm{fcc}}$ correspond to the reciprocal lattice points $(0,0,0)$ and $(2 \pi / \mathrm{a}, 0,0)$, respectively. Panels $(\mathrm{b}-\mathrm{f})$ only show the first $20 \%$ of the full $\Gamma-\mathrm{X}_{\mathrm{fcc}}$ range, with the axis truncated at $0.2 \times(2 \pi / \mathrm{a}, 0,0)$.

minima split into two sets: a two-fold degenerate band with minima at the $\Gamma$ point, and four $1 \Delta$ bands with degenerate minima at approximately $0.85 \times 2 \pi /$ a along the four equivalent in-plane $\Gamma-\mathrm{X}_{\mathrm{fcc}}$ directions. In this work we will generally utilise in-plane repeats that are larger than one atom which causes the band structure to fold into correspondingly smaller Brillouin zones as illustrated in Fig. 1(f-h). Specifically, in unit cells with a 2 -atom repeat and larger (up to a 16-atom repeat), the $1 \Delta$ band minima is shifted to approximately $0.15 \times 2 \pi /$ a . This is shown for an 8-atom repeat in Fig. 2(b).

Figure 2(c) illustrates the effects of introducing phosphorus dopants $(1 / 4 \mathrm{ML})$ into the $\delta$-plane using our mixed-atom pseudopotential representation. Doping creates an attractive potential which pulls down several conduction bands (principally $1 \Gamma, 2 \Gamma$ and $1 \Delta$ ) into the silicon band-gap. These bands are populated by the donor electrons, creating a two-dimensional electron gas (2DEG). The lowest two levels, $1 \Gamma$ and $2 \Gamma$, are split by a small amount $(0.006 \mathrm{eV})$. This energy difference is commonly referred to as the valley splitting.

The mixed-atom pseudopotential artificially averages the distribution of phosphorus atoms in the dopant plane; this acts to lessen the confinement of the donor electrons and hence the valley splitting is rather small. ${ }^{20}$ Employing an explicit representation of dopant atoms (i.e. dis- tinct silicon and phosphorus atoms) increases the confinement with a strong effect on the band structure. This is illustrated in Fig. 2(d) for a highly-ordered arrangement of phosphorus atoms with a 4 -atom repeat (highlighted by a gray background in the dopant plane schematic). We see that: (i) the near-degenerate $1 \Gamma / 2 \Gamma$ bands in the mixed-atom treatment become widely separated, leading to much larger valley splitting of $0.153 \mathrm{eV}$, and (ii) the $1 \Gamma$ and $1 \Delta$ bands, which are non-interacting in the mixed-atom case, undergo an avoided crossing. It should be emphasized that the 4-atom dopant arrangement is almost certainly too ordered as there is no experimental evidence that phosphorus dopants adopt any degree of ordering in the $\delta$-plane.

The simplest way to represent disorder using an explicit treatment is to use a larger repeat unit that contains eight atoms in the plane, two of which are phosphorus. These phosphorus atoms are positioned nearest each other in the dopant plane, corresponding to nextnearest neighbours in the bonding network; Fig. 2(e) shows the band structure for this arrangement. Qualitatively, the band structure is similar to that of the mixedatom pseudopotential, with well-defined $1 \Gamma, 2 \Gamma$ and $1 \Delta$ bands present in the band gap. Common to both is the absence of an avoided crossing between the $1 \Gamma$ and $1 \Delta$ levels. Where the two band structures differ is in the val- 

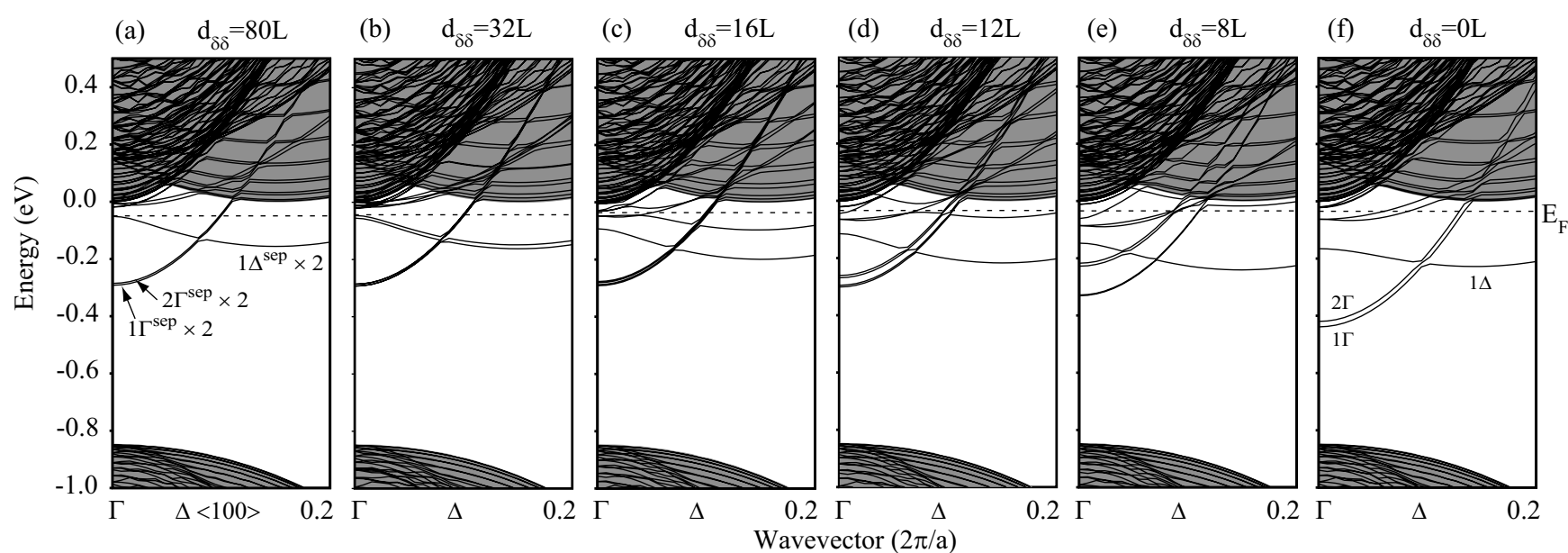

FIG. 3: Bandstructures for two interacting phosphorus $\delta$-doped layers (1/4 ML) at separations between 80 and $0 \mathrm{~L}$. Calculations were performed using mixed-atom pseudopotentials in a $200 \mathrm{~L}$ unit cell with a 2 -atom in-plane repeat. Only the first $20 \%$ of the $\Gamma-\mathrm{X}_{\mathrm{fcc}}$ range is shown, with the axis truncated at $0.2 \times(2 \pi / \mathrm{a}, 0,0)$.

ley splitting, with the 8-atom explicit-disordered structure exhibiting a pronounced separation $(0.081 \mathrm{eV})$, much larger than that of the mixed-atom $(0.006 \mathrm{eV})$ and around half that of the 4-atom explicit-ordered system $[0.153 \mathrm{eV}$; $c f$. Fig. 2(d)]. These features of the 8-atom band structure quantitatively match those of an even larger disordered arrangement with a 16-atom repeat for which the valley splitting is $0.076 \mathrm{eV}$ (cf. Figs. 10(c,d) in Ref. 20).

While economic, the SZP basis set is associated with errors in the band energies as has been previously described for bulk silicon [see Table 1 in Ref. 20] and an ordered 4-atom repeat of phosphorus in $\delta$-doped silicon. ${ }^{21}$ Figure 2(f) shows the band structure of the 8-atom explicit-disordered system calculated using a more complete DZP basis set. Qualitatively, the DZP and SZP band structures are very similar, with the largest difference being the smaller bulk silicon band-gap. For the valley splitting we calculate a value of $0.063 \mathrm{eV}$ with a DZP basis, $0.018 \mathrm{eV}$ smaller than the SZP case. This DZP value is our best estimate for the valley splitting in a realistically disordered $1 / 4$ ML $\delta$-layer within a DFTGGA framework.

The mixed-atom, explicit-ordered and explicitdisordered representations as well as the SZP and DZP treatments each have their own distinct advantages and disadvantages. The mixed-atom treatment is appealing due to the conceptual simplicity of its band structure and the much reduced computational cost. The two explicit representations provide a more rigorous treatment of the band structure (principally the valley splitting), but this gain comes at considerable computational cost due to the large in-plane repeats required. Of the two explicit-atom treatments, explicit-disorder provides the best description of realistically disordered structures, but the computational cost makes this high-level treatment unsuitable for systematic investigations. A similar balance governs the choice between SZP and DZP basis sets: SZP offers numerical stability and computational speedups, while the DZP treatment provides band energies which are closer to the basis-set converged limit, ${ }^{21}$ albeit at considerable computational cost. One of the challenges in this work is to balance these three levels of approximations, namely mixed-atom versus explicit-atom, ordered versus disordered dopants, and SZP versus DZP bases. Here we employ all three approaches in various combinations. We first use the mixed-atom approach with an SZP basis to qualitatively describe the physics of two interacting $\delta$-layers. This is followed in turn by a refined treatment at selected, critical distances using explicit dopants in both ordered and disordered arrangements. Lastly, we assess the effects of a full DZP treatment on our results.

\section{B. Double $\delta$-layers: mixed-atom representation}

We start by describing the interaction of two $1 / 4 \mathrm{ML}$ $\delta$-layers using a large $200 \mathrm{~L}$ unit cell, a 2-atom in-plane repeat and the mixed-atom pseudopotential approach. Figure 3 shows the progression of the band structure as the $\delta$-layer separation, $\mathrm{d}_{\delta \delta}$, is reduced from $80 \mathrm{~L}[$ Fig. 3(a)] to 0L [Fig. 3(f)]. At 80L, the two $\delta$-layers are effectively separated. This is evident in the fact that the band structure is virtually identical to that of the corresponding single layer structure [cf. Fig. 2(c)], except that all bands are doubled. As $\mathrm{d}_{\delta \delta}$ is reduced to 32 and $16 \mathrm{~L}$ [Figs. 3(b,c), respectively], the pair of $1 \Delta$ bands split apart, indicating that the interaction between the two dopant layers commences with these bands. At 12L separation [Fig. 3(d)], a splitting of the lower lying $\Gamma$ levels becomes apparent as well. By 8L [Fig. 3(e)], the upper branch of the $1 \Delta$ pair has almost merged with the bulk CBM and the interacting $\Gamma$ bands have split by more than $0.1 \mathrm{eV}$. The last panel [Fig. 3(f)] shows the two layers completely merged into 


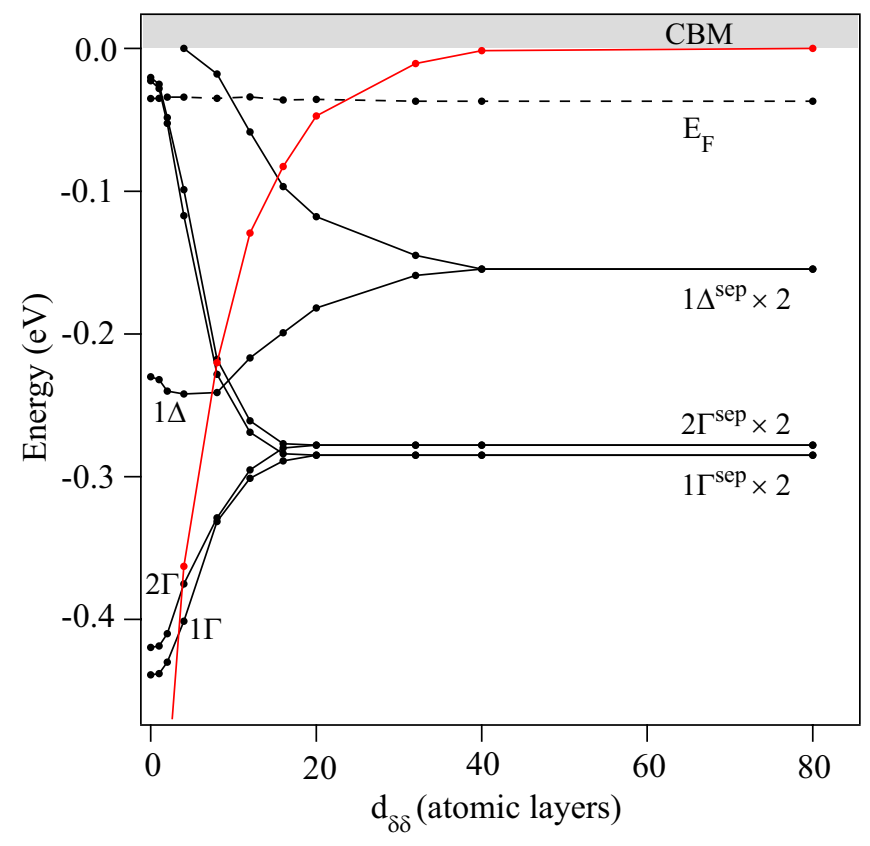

FIG. 4: Band minima for $1 \Gamma, 2 \Gamma, 1 \Delta$ and $\mathrm{E}_{\mathrm{F}}$ as a function of the separation $\left(\mathrm{d}_{\delta \delta}\right)$ between two $1 / 4 \mathrm{ML} \delta$-doped layers. The red line presents the plane average of the doping potential at the halfway point between the two $\delta$-layers. Calculations were performed using mixed-atom pseudopotentials in a $200 \mathrm{~L}$ cell.

a single $1 / 2$ ML $\delta$-layer. This $1 / 2$ ML layer exhibits all the band structure characteristics of the isolated $1 / 4 \mathrm{ML}$ layer, except that the $1 \Gamma / 2 \Gamma$ and $1 \Delta$ levels are pulled deeper into the band gap due to the stronger confinement generated by $1 / 2$ ML phosphorus dopants.

With Fig. 4 we consider the same system but focus on the band minima for a larger set of separations $\mathrm{d}_{\delta \delta}$. Also plotted in Fig. 4 is the Fermi level, $\mathrm{E}_{\mathrm{F}}$, and the planeaverage of the doping potential halfway between the two $\delta$-layers. Looking first at the band minima, the figure further highlights the observations made above. We see that the $1 \Delta$ bands begin to split at separations below $40 \mathrm{~L}$, whereas the $1 \Gamma$ and $2 \Gamma$ bands only interact at separations below 20L. The data shows how one interaction branch is stabilised, evolving into the $1 \Delta, 1 \Gamma$ and $2 \Gamma$ band minima of the merged, $1 / 2$ ML system, while the other branch becomes rapidly destabilised. At very close separations, $\mathrm{d}_{\delta \delta}$ less than $2 \mathrm{~L}$, the band minima flatten out. These separations no longer correspond to a pair of $\delta$-layers, but rather represent a single layer with a degree of vertical dopant disorder. Such disorder is known ${ }^{16,18}$ to have little effect on the band energies provided the vertical dopant distribution is small in comparison to the width of the doping potential.

Figure 5 shows the plane-averaged donor density and doping potential at four $\delta$-layer separations $(0,12,32$ and $80 \mathrm{~L}$ ). The doping potential (red line in Fig. 5) is calculated as the difference between the electrostatic potentials of $\delta$-doped and undoped supercells. At a separation of $80 \mathrm{~L}$ the doping potential is effectively the sum

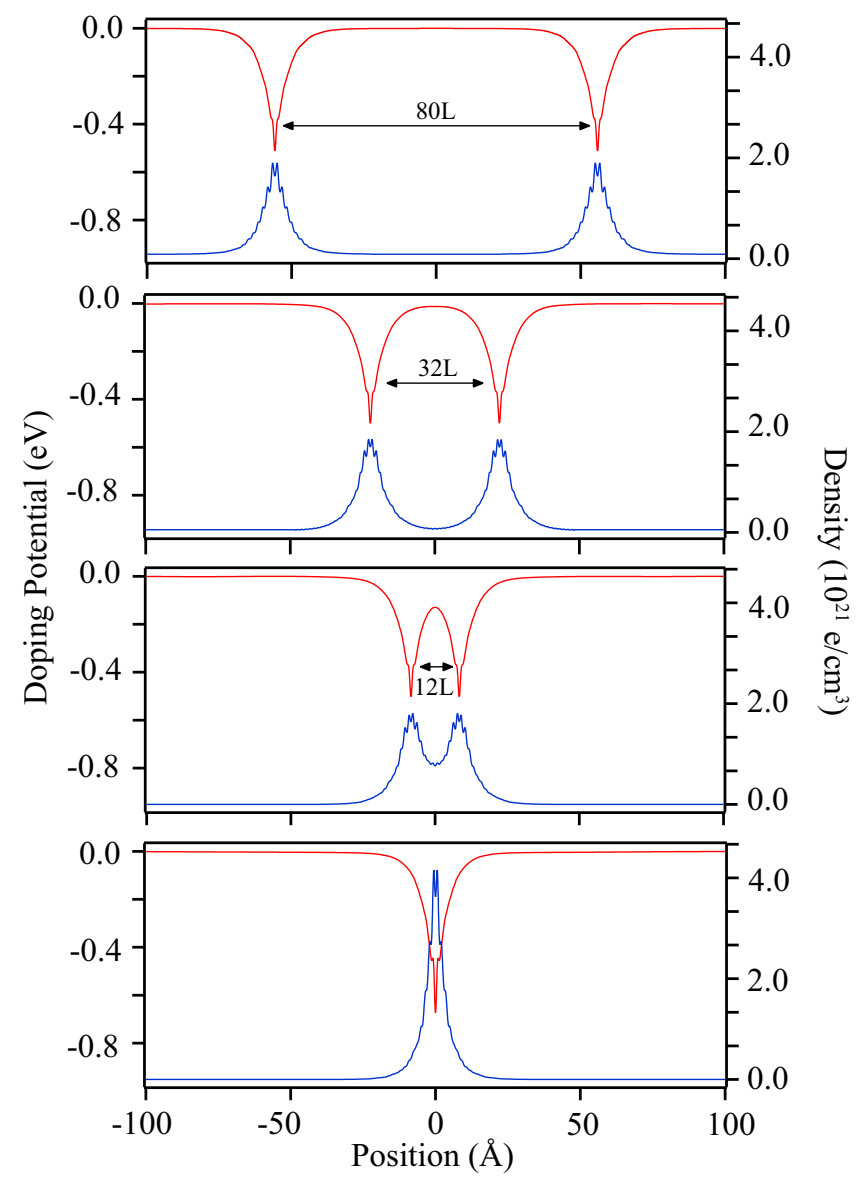

FIG. 5: Donor density (blue line) and doping potential (red line) for two $1 / 4 \mathrm{ML}$ phosphorus $\delta$-doped layers, at separations of (a) 80, (b) 32, (c) 12 and (d) 0 silicon layers (L). Calculations were performed using mixed-atom pseudopotentials in a 200L cell.

of two non-interacting potentials, each of which exhibits the well-known V-shaped appearance characteristic of single $\delta$-layers. The independence of the two $\delta$-layers at this separation is reflected in the fact that the doping potential at the midpoint plane is essentially zero $(<0.05 \mathrm{meV})$. As the $\delta$-layers approach, the tails of the individual potentials begin to overlap, lowering the midpoint potential to -0.011 and $-0.129 \mathrm{eV}$ at separations of 32 and $12 \mathrm{~L}$, respectively. At $0 \mathrm{~L}$ the potentials have completely merged into a single $\mathrm{V}$-shaped potential at the mid-point. The donor density distribution (blue line in Fig. 5) was calculated by integrating over all bands between the bulk valence band maximum and the Fermi energy. The evolution of the donor density closely mirrors the dopant potential. With decreasing separation the midpoint donor density is seen to gradually increase, illustrating the merging of two isolated 2DEG's into one.

It is instructive to correlate the evolution of the midpoint potential with that of the band minima. This comparison is made in Fig. 4 where the midpoint potential is included as a red line. It can be seen that the devia- 
tion of the midpoint potential from zero at a separation of around $32 \mathrm{~L}$ is associated with the onset of splitting of the two degenerate $1 \Delta$ levels. Only at smaller separations, around $16 \mathrm{~L}$, does the lowering of the midpoint potential lead to splitting of the $1 \Gamma / 2 \Gamma$ levels. Note that the midpoint potential and band minima are plotted on the same scale, demonstrating that the depth of the potential is similar in magnitude to the band-splitting arising from interactions between $\delta$-layers.

Our results thus far have a number of points of contact with earlier theoretical studies in the literature. Rodriguez-Vargas and Gaggero-Sager ${ }^{23}$ used a ThomasFermi model to describe interacting pairs of $n$-type $\delta$ doped layers in silicon. For three different dopant concentrations $(1 / 85,1 / 52$ and $1 / 10 \mathrm{ML})$, they describe the interlayer splitting as function of separation. Qualitatively, their diagrams are similar to our Fig. 4, with higher lying bands interacting at larger separations than lower lying bands. Due to the lesser confinement at these lower dopant concentrations the layers begin interacting at considerably larger separations (180L for $1 / 85 \mathrm{ML}$ ) as compared to our high-density $1 / 4 \mathrm{ML}$ case (onset below $40 \mathrm{~L}$ as discussed above). The low concentrations considered by Rodriguez-Vargas and Gaggero-Sager correspond to the donor densities achieved when $\delta$-layers are fabricated by molecular-beam-epitaxy techniques. ${ }^{31} \mathrm{We}$ instead, focus on the high-density (1/4 ML), atomically sharp $\delta$-layers that can be fabricated by phosphine dissociation and thermal incorporation. ${ }^{3,4,24}$ Relevant also are two theoretical studies which have considered $\delta$-layer interactions in periodic superlattices. Scolfaro et al. ${ }^{13}$ used effective mass theory to study a wide range of dopant concentrations and periodic repeat lengths. Their data shows that with increasing dopant concentration the onset of splitting commences at shorter superlattice repeats. For example, at a doping concentration of $1 / 52 \mathrm{ML}$ splitting of the longitudinal $(\Delta)$ level commences at a periodic repeat of approximately 110L, while at $1 / 10$ ML splitting commences around $70 \mathrm{~L}$. These values follow a trend that is compatible with our values at $1 / 4 \mathrm{ML}$. Scolfaro et $a l .{ }^{13}$ also report in their Fig. 4 high dopant concentration data for a periodic repeat of $50 \AA$ (i.e. $37 \mathrm{~L}$ ). Our $1 / 4 \mathrm{ML}$ calculations (corresponding to a donor concentration of $1.7 \times 10^{14} \mathrm{~cm}^{-2}$ ) are consistent with their data in that at a $40 \mathrm{~L}$ separation we see no splitting of the transverse $(\Gamma)$ band and minimal splitting of the longitudinal $(\Delta)$ band. Recent DFT calculations reported by Drumm et $a l .{ }^{21}$ studied $1 / 4$ ML $\delta$-layers, with a particular question being the number of silicon layers required to electronically separate a $\delta$-layer from its periodic image. They report well-separated results for 80L supercells, and small band-shifts (around $0.04 \mathrm{eV}$ ) at $40 \mathrm{~L}$. This too is consistent with our result (Fig. 4), keeping in mind that superlattice interactions commence at slightly longer range than pair interactions.

\section{Double $\delta$-layers: explicit dopant representation}

The mixed-atom pseudopotential model is a simplified treatment which neglects the discrete nature of the dopant atoms. To quantify this effect, we considered two characteristic separations, $32 \mathrm{~L}$ and $12 \mathrm{~L}$, using an explicit dopant model. As discussed above, these separations are just within the onset of interlayer splitting in the $1 \Delta$ and $1 \Gamma / 2 \Gamma$ bands, respectively. Use of explicit-atom doping requires us to define the precise arrangement of dopant atoms in the plane and their relative registry from one plane to the next. Figure 6 compares for these two separations the band structure of four explicit-atom arrangements with two mixed-atom calculations. The arrangement of the phosphorus atoms in each layer is illustrated at the top. With the exception of the right-most panels in Fig. 6, all of the calculations employ an SZP basis. The first three of these explicit dopant structures (i.e. left half of Fig. 6) employ an ordered 4-atom pattern in both $\delta$-layers, differing only in the relative registration of the phosphorus atoms between the two planes. The fourth explicit dopant structure employs the quasi-disordered 8-atom pattern in each layer. The SZP-basis mixedatom pseudopotential calculations [Figs. 6(e,k)] are included for reference; the DZP-basis calculations of the same structure are discussed in the following Section.

We consider first a separation distance of 32L [see top row of panels, (a)-(f), in Fig. 6]. As seen earlier, the $1 \Delta$ band in the mixed-atom pseudopotential [Figs. 3(b) \& 6(e)] exhibits a small interlayer splitting of $0.015 \mathrm{eV}$, while the $1 / 2 \Gamma$ bands remain unaltered. In the corresponding explicit-dopant structures [Figs. $6(\mathrm{a}-\mathrm{c})]$ a much larger valley splitting occurs, against which the splitting due to the interlayer interaction is dwarfed. This contrast between the valley and interlayer splitting is most clearly seen for the explicit-disordered case [Fig. 6(d)] that has no avoided crossing. For the three ordered arrangements the interlayer splitting is only apparent in those bands around the avoided crossing which have $1 \Delta$ character. The lowest (adiabatic) band in Figs. 6(a-c) is nearly degenerate around the $\Gamma$ point, with a small splitting emerging as it mixes with the diabatic $1 \Delta$ band. The splitting is also evident to the left of the avoided crossing (i.e. closer to the $\Gamma$ point) in one of the higher bands close to the Fermi level. We note that the three registries of the ordered 4-atom patterns [Figs. 6(a-c)] give rise to virtually identical band structures, showing that at this separation distance the relative placement of dopants between the two layers has little effect on the electronic structure. Returning to the explicit-disordered case [Fig. 6(d)], we observe an interlayer splitting (ILS) of the $1 \Delta$ band of $0.014 \mathrm{eV}$. As seen in Table I, almost exactly the same value is obtained for the mixed-atom pseudopotential, highlighting the utility of the mixedatom representation. The valley splitting (VS) for the explicit-disordered case is $0.081 \mathrm{eV}$ which is significantly smaller than in the explicit-ordered cases, in line with the reduction seen for the single-layer band structure (Fig. 2). 


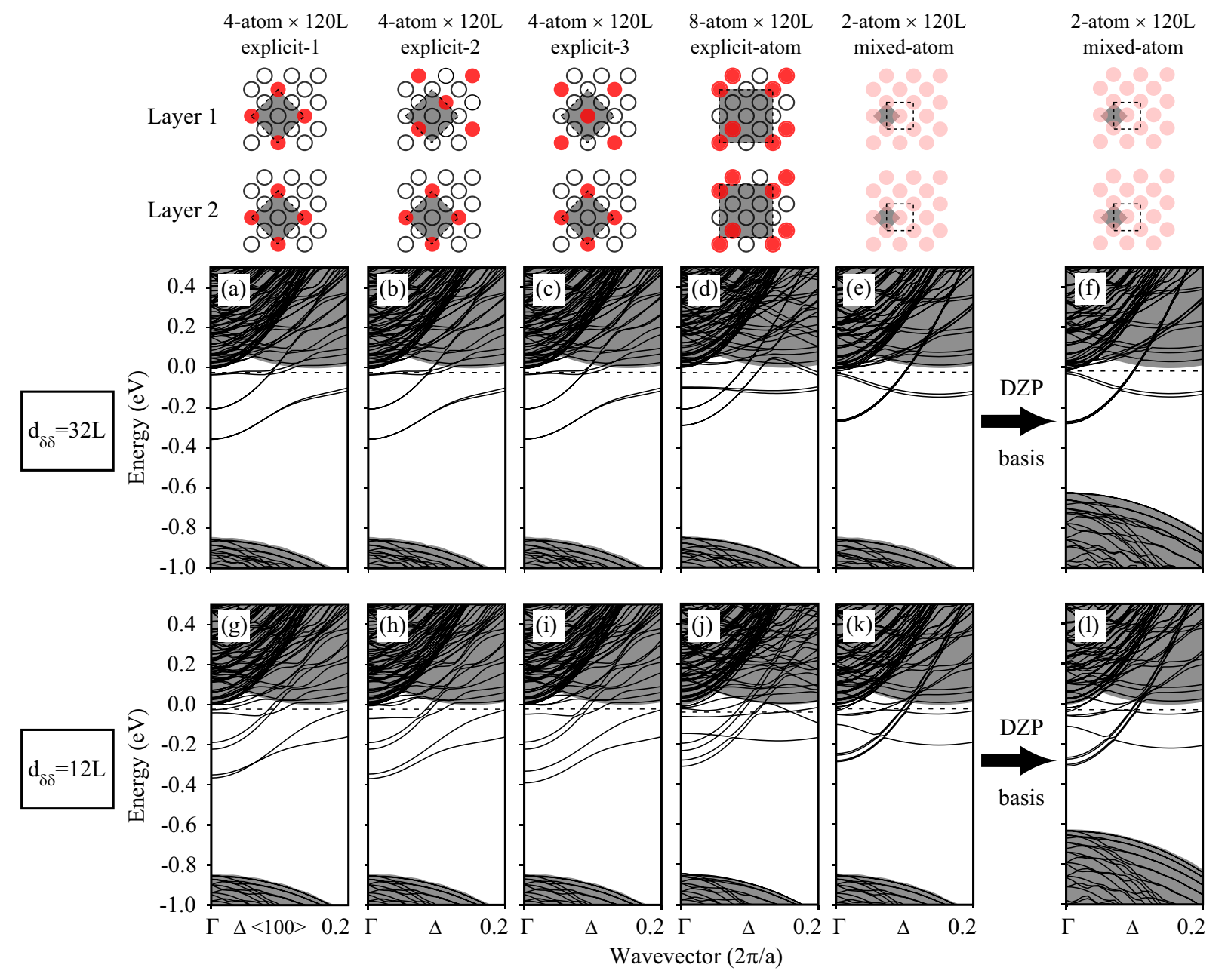

FIG. 6: Band structures for 1/4 ML double $\delta$-layers, at separations of 32 and 12 layers (L), using four explicit representations and the mixed-atom pseudopotential. The schematic diagrams in the top row illustrate the different atomic representations within the dopant planes, with the gray square denoting the in-plane unit cell. With the exception of panels (f) and (l) which were computed using a DZP basis, all calculations were performed using an SZP basis. Only the first $20 \%$ of the $\Gamma-\mathrm{X}_{\mathrm{fcc}}$ range is shown, with the axis truncated at $0.2 \times(2 \pi / \mathrm{a}, 0,0)$.

At a separation distance of $12 \mathrm{~L}$, both the $1 \Gamma / 2 \Gamma$ and $1 \Delta$ bands participate in the interaction between layers. In the band structure, the effect of explicit doping is most easily appreciated by comparing the explicitdisordered case [Fig. 6(j)] with the mixed-atom pseudopotential in Fig. $6(\mathrm{k})$. In both cases the $1 \Delta$ band undergoes a large splitting (0.141 and $0.154 \mathrm{eV}$, respectively; see Table I) with the upper branch almost merging with the conduction band. Table I further shows that for the $1 \Gamma / 2 \Gamma$ bands the interlayer splitting in the explicit-disordered and mixed-atom cases is very similar, being 0.034 and $0.033 \mathrm{eV}$, respectively. ${ }^{32}$ The valley splitting in the explicit-disordered case is much larger than in the mixed-atom case (0.082 and $0.007 \mathrm{eV}$, respectively). As a consequence, with the mixed-atom pseudopotential the interlayer splitting is larger than the valley splitting, while for the explicit-disordered structure the converse occurs. The largest valley splittings are seen for the three explicit-ordered cases [Figs. 6(g-i)] where the band structures are complicated by the $1 \Gamma / 1 \Delta$ avoided crossing. Here the interlayer splitting again varies according to the degree of adiabatic mixing, with the lowest bands transitioning from a smaller splitting associated with the diabatic $1 \Gamma$ band to a larger splitting of the $1 \Delta$ band. In contrast, the $2 \Gamma$ band, which does not participate in the avoided crossing, exhibits a more uniform interlayer splitting. We note in passing that at this shorter separation the avoided crossing is more sensitive to the registry of the ordered dopant planes, with the bands being slightly shifted relative to each other. We reiterate, however, that this type of ordering is unlikely to be present in experimentally prepared double $\delta$-layers, and thus the explicit-disordered case in Figs. 6(d,j) provide the most realistic representation of the double $\delta$-layer band structure at the SZP level. 
TABLE I: Valley splitting (VS) and interlayer splitting (ILS) energies for single and double $\delta$-layers using mixed-atom and explicit-disordered representations. All calculations are performed with an SZP basis, and energies are reported in $\mathrm{eV}$. Splitting for the double $\delta$-layer systems are averages of the $1 / 2 \Gamma$ bands. ${ }^{32}$ The $1 \Delta$ splittings for the $12 \mathrm{~L}$ are vertical bandenergy differences measured from the minimum in the lower branch.

\begin{tabular}{lcccc}
\hline \hline & & \multirow{2}{*}{$1 \times \mathrm{Si}: \mathrm{P}-\delta$} & \multicolumn{2}{c}{$2 \times \mathrm{Si}: \mathrm{P}-\delta$} \\
& & & $(32 \mathrm{~L})$ & $(12 \mathrm{~L})$ \\
\hline VS & Mixed & 0.006 & 0.007 & 0.007 \\
& Explicit & 0.081 & 0.081 & 0.082 \\
ILS $(\Gamma)$ & Mixed & & $<0.001$ & 0.033 \\
& Explicit & & $<0.001$ & 0.034 \\
ILS $(\Delta)$ & Mixed & & 0.015 & 0.154 \\
& Explicit & & 0.014 & 0.141 \\
\hline \hline
\end{tabular}

\section{Hybrid approach for double $\delta$-layers}

A full DZP treatment of an explicitly disordered double $\delta$-layer system lies beyond the computational reach of this work; however, examination of Table I suggests a hybrid strategy for estimating the valley splitting and two interlayer splittings in the DZP regime. This strategy is based on two important observations from our SZP calculations: (i) the valley splitting of interacting double $\delta$-layers is the same as that of individual $\delta$-layers, and (ii) the interlayer splitting of the $\Gamma$ and $\Delta$ bands is the same in both the mixed-atom and explicit-atom representations. Accordingly, we can confidently estimate the DZP valley splitting in the double $\delta$-layer case by performing a single $\delta$-layer calculation using a quasi-disordered explicit-atom dopant arrangement with a DZP basis [see Fig. 2(f)]. We can similarly estimate the DZP interlayer splitting by performing a mixed-atom calculation for a double $\delta$-layer system with a DZP basis [Figs. 6(f,l)]. Both of these calculations are computationally tractable, whereas a DZP calculation of a quasi-disordered double $\delta$-layer system is not.

Our best estimates for the interlayer and valley splitting at separations of $32 \mathrm{~L}$ and $12 \mathrm{~L}$ are given in Table II, with the corresponding mixed-atom band struc-

TABLE II: Best estimates for the valley splitting (VS) and interlayer splitting (ILS) using our hybrid approach. All energies are in $\mathrm{eV}$.

\begin{tabular}{lcccc}
\hline \hline & \multirow{2}{*}{$1 \times \mathrm{Si}: \mathrm{P}-\delta$} & \multicolumn{2}{c}{$2 \times \mathrm{Si}: \mathrm{P}-\delta$} \\
& & $(32 \mathrm{~L})$ & $(12 \mathrm{~L})$ \\
\hline VS & Explicit & 0.063 & & \\
$\operatorname{ILS}(\Gamma)$ & Mixed & & $<0.001$ & 0.035 \\
$\operatorname{ILS}(\Delta)$ & Mixed & & 0.017 & 0.169 \\
\hline \hline
\end{tabular}

tures shown in Figs. 6(f,l). For the valley splitting, the double layer systems have the same (average) splitting as the single layer case. The only caveat is for extremely close separations at which the two layers are effectively merged into a single layer at twice the concentration. For the interlayer splittings, all of our best-estimate values are larger (by roughly $5-10 \%$ ) than their SZP equivalents in Table I, with the obvious exception of $\operatorname{ILS}(\Gamma)$ at $32 \mathrm{~L}$ which is negligible in each case. This observation of increased interlaying splitting with a DZP basis can be intuitively interpreted by considering the width of the donor density distribution in single $\delta$-layers; about $15 \%$ larger with a DZP basis than with SZP. ${ }^{21}$ Accordingly, the increased interlayer splitting is completely consistent with the traditional overlap model from molecular orbital theory in which splitting of atomic energy levels correlates with the spatial extent of the free-atom wavefunctions and the associated bond integral (see Ref. 33).

\section{CONCLUSION}

In summary, we have used a density functional theory model to examine changes in the electronic structure of phosphorus $\delta$-doped layers in silicon as the distance between $\delta$-doped layers is altered. Using a combination of mixed-atom and explicit-atom pseudopotentials we showed that the band structure is largely unchanged for separations above 40 layers (40L). For separations below $40 \mathrm{~L}$ the $1 \Delta$ band undergoes splitting which increases in magnitude as the separation decreases. Splitting of the $\Gamma$ bands is only seen for separations less than $16 \mathrm{~L}$. We show that the mixed-atom representation provides a useful approximation to an explicit-atom system with a quasi-disordered arrangement of dopants in the $\delta$-plane. In an unexpected finding, we observed that the interlayer splitting of the mixed-atom and explicit-atom representations are virtually identical. Coupled with the observation that the valley splitting in double $\delta$-layer system is the same as single $\delta$-layers, this opens up a hybrid strategy for estimating double $\delta$-layer energy splittings of DZP-basis quality without having to perform the full explicit-atom quasi-disordered calculation. The hybrid strategy provides a recipe for future work, enabling the calculation of double $\delta$-layer splittings at arbitrary separations and dopant concentrations.

\section{Acknowledgements}

We thank Justin Wells for helpful discussions. This work was supported by the Australian Research Council through the Centre of Excellence for Quantum Computation and Communication Technology (Project No. CE1100096). The project used computational resources provided by the Australian National Computational Infrastructure (NCI) and the iVEC facility at Murdoch University. 
1 S. R. Schofield, N. J. Curson, M. Y. Simmons, F. J. Rueß, T. Hallam, L. Oberbeck, and R. G. Clark, Phys. Rev. Lett. 91, 136104 (2003).

2 F. J. Rueß, W. Pok, T. C. G. Reusch, M. J. Butcher, K. E. J. Goh, L. Oberbeck, G. G. Scappucci, A. R. Hamilton and M. Y. Simmons, Small 3, 563 (2007).

3 T. C. Shen, J. Y. Ji, M. A. Zudov, R. R. Du, J. S. Kline, and J. R. Tucker, Appl. Phys. Lett. 80, 1580 (2002).

${ }^{4}$ L. Oberbeck, N. J. Curson, M. Y. Simmons, R. Brenner, A. R. Hamilton, S. R. Schofield, and R. G. Clark, Appl. Phys. Lett. 81, 3197 (2002).

${ }^{5}$ K. E. J. Goh, L. Oberbeck, M. Y.Simmons, A. R. Hamilton, and M. J. Butcher, Phys. Rev. B 73, 035401 (2006).

${ }^{6}$ S. J. Robinson, J. S. Kline, H. J. Wheelwright, J. R. Tucker, C. L. Yang, R. R. Du, B. E. Volland, I. W. Rangelow, and T.-C. Shen, Phys. Rev. B 74, 153311 (2006).

7 A. Fuhrer, M. Füchsle, T. C. G. Reusch, B. Weber, and M. Y. Simmons, Nano. Lett. 9, 707 (2006).

8 B. Weber, S. Mahapatra, H. Ryu, S. Lee, A. Fuhrer, T. C. G. Reusch, D. L. Thompson, W. C. T. Lee, G Klimeck, L. C. L. Hollenberg, and M. Y. Simmons, Science 335, 64 (2012).

9 M. Fuechsle, S. Mahapatra, F. A. Zwanenburg, M. Friesen, M. A. Eriksson, and M. Y. Simmons, Nat. Nanotechnol. 5, 502 (2010).

10 M. Fuechsle, J. A. Miwa, S. Mahapatra, H. Ryu, S. Lee, O. Warschkow, L. C. L. Hollenberg, G. Klimeck, and M. Y. Simmons, Nat. Nanotechnol. 7, 242 (2012).

11 S. R. McKibbin, W. R. Clarke, A. Fuhrer, T. C. G. Reusch, and M. Y. Simmons, Appl. Phys. Lett. 95233111 (2009).

12 S. R. McKibbin, W. R. Clarke, A. Fuhrer, and M.Y. Simmons, J. Cryst. Growth 312, 3247 (2010).

13 L. M. R. Scolfaro, D. Beliaev, R. Enderlein, and J. R. Leite, Phys. Rev. B 50, 8699 (1994).

14 D. W. Drumm, L. C. L. Hollenberg, M. Y. Simmons, and M. Friesen, Phys. Rev. B 85, 155419 (2012).

15 G. Qian, Y.-C. Chang, and J.R. Tucker, Phys. Rev. B 71, 045309 (2005).

16 X. Cartoixà and Y.-C. Chang, Phys. Rev. B 72, 125330 (2005).

17 H. Ryu, S. Lee, and G. Klimeck, IEEE Proceedings of the 13th International Workshop on Computational Electron- ics, Tsinghua University, Beijing 10, 21 (2009).

18 S. Lee, H. Ryu, H. Campbell, L. C. L. Hollenberg, M. Y. Simmons, and G. Klimeck, Phys. Rev. B 84, 205309 (2011).

19 D. J. Carter, O. Warschkow, N. A. Marks, and D. R. McKenzie, Phys. Rev. B, 79, 033204 (2009); ibid. 80, 049901 (2009).

20 D. J. Carter, O. Warschkow, N. A. Marks, and D. R. McKenzie, Nanotechnol. 22, 065701 (2011).

21 D. W. Drumm, A. Budi, M. C. Per, S. P. Russo, and L. C. L Hollenberg, arXiv:1201.3751v1.

22 A. Budi, D. W. Drumm, M. C. Per, A. Tregonning, S. P. Russo, and L. C. L Hollenberg, Phys. Rev. B 86, 165123 (2012).

23 I. Rodriguez-Vargas and L. M. Gaggero-Sager, J. Appl. Phys. 99, 033702 (2006).

${ }^{24}$ H. F. Wilson, O. Warschkow, N. A. Marks, N. J. Curson, S. R. Schofield, T. C. G. Reusch, M. W. Radny, P. V. Smith, D. R. McKenzie, and M. Y. Simmons, Phys. Rev. B 74, 195310 (2006).

25 J. M. Soler, E. Artacho, J. D. Gale, A. Garcia, J. Junquera, P. Ordejon, and D. Sanchez-Portal, J. Phys.:Condens. Matter 14, 2745 (2002).

26 N. Troullier and J. L. Martins, Phys. Rev. B 43, 1993 (1991).

27 J. P. Perdew, K. Burke, and M. Ernzerhof, Phys. Rev. Lett. 77, 3865 (1996).

28 L. Nordheim, Ann. Phys. (Leipzig) 9, 607 (1931).

29 L. Bellaiche and D. Vanderbilt, Phys. Rev. B 61, 7877 (2000).

30 M. Methfessel and A. T. Paxton, Phys. Rev. B 40, 3616 (1989)

31 H. P. Zeindl, T. Wegehaupt, I. Eisele, H. Oppolzer, H. Reisinger, G. Tempel, and F. Koch, Appl. Phys. Lett. 50, 1164 (1987).

32 For simplicity, the splittings are expressed as averages. For example, the individual interlayer splittings for $1 \Gamma$ and $2 \Gamma$ are 0.031 and $0.037 \mathrm{eV}$ for the explicit-disordered case, and 0.032 and $0.034 \mathrm{eV}$ for the mixed-atom case.

33 D. G. Pettifor, Bonding and structure of molecules and solids, (Oxford University Press, Oxford, 2002). 